\section{Military Technical College Kobry El-Kobbah, Cairo, Egypt.}

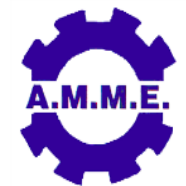

\title{
HIGH ACCURACY SPACECRAFT ORBIT PROPAGATOR VALIDATION
}

\author{
A. Refaat ${ }^{1}$, A. Badawy ${ }^{2}$, M. Ashry ${ }^{1}$ and Adel Omar ${ }^{1}$
}

\begin{abstract}
The Space Situational Awareness (SSA) problem is becoming an exponentially significant concern for satellite operators. The threat of on-orbit collisions endangers satellites, spacecrafts and astronauts themselves especially in low-earth orbits (LEO). Many collisions occurred between satellites and debris that include natural, operational and non-operational objects in space. The collision between Russian cosmos 2251 and American iridium 33, for instance, imposed the need for an accurate orbital propagation module. In order to perform successful collision avoidance maneuvers, the mission operators need the orbit prediction to be highly accurate. Simple impractical number of collision avoidance maneuvers represents dreadful solution to escape of collision. Fake warning means waste of fuel and resources of satellite. This paper introduces an enhanced model to increase the accuracy of propagation estimation based on a precise perturbation model.
\end{abstract}

\section{KEY WORDS}

Orbit, propagation, drag, TLE, Cowell, STK, SGP4, hpop, eclipse, ephemerides. 


\section{INTRODUCTION}

Mission control center planning would have incorrect procedures for collision analysis specially for LEO orbits where a spatial density about $4.8 \times 10^{-8} \mathrm{object} / \mathrm{km}^{3}$ for objects larger than $10 \mathrm{~cm}[1]$, as a result of following an inaccurate orbit prediction method and may lead to actual collision. The source of knowledge of other objects is the public two-line element set (TLEs). But care should be taken because propagations using TLEs is based on the simplified general perturbations (SGP4). Consequently, it is not accurate enough to be the base of judgment for maneuvers. The problem now is that both objects are subjected to these inaccurate predictions.

The model proposed here is based on Cowell's method through integration the acceleration of two bodies beside the perturbed accelerations. The results are then compared to those using with SGP4 and real data. However, the accuracy of TLEs and related SGP4 should be considered to judge the level of confidence for these data. Several studies have compared SGP4 with real data as [2].A comparison between GPS satellites positions and its position calculated by SGP4 is conducted. The results show that the range error for around 14 days is about $35-45 \mathrm{~km}$.

Several studies have been carried out attempting to enhance the propagation using Cowell's method [3],They median orbit propagator including 60x60 EGM2008 gravity model and MSISE 1990 for atmospheric drag model and solar radiation pressure using simple biconic approximation and assuming fixed values for the solar radio flux (F10.7) and disturbances in the horizontal component of earth's magnetic field (KP).

Multiple TLEs are used for forward propagation from each TLE to the next then propagate backward for each sequence TLE with linear systematic bias approximation with SGP4 as propagator but the results reach about $4 \mathrm{~km}$ in 7 days moreover it needs a sequence of TLEs so it can't be applied to current satellite it must be old trajectory for available data [4].

Muldoon et. al. also used a sequence of TLE with naïve modeling approaches with blending for 411 debris, but he found large errors on objects with large deviation due to the noise in mean motion and epoch time and this is the nature of motion in space with environment changes non-periodically [5].

European space agency developed techniques based on TLEs to get the initial state at certain epoch. TLE data are then followed by some numerical propagation of the orbit determination result using force models, a testing of the SGP4 is done by Easthope [6].

An accurate model prediction called SpOCK by Charles-Virat [7], but they used EGM96 as earth gravitational model, also Runge kutta of the fourth order with fixed step size is used for the integration, and also NRLSMSIS-00e for atmospheric density at the position of spacecraft moreover he made the comparison for only one day with the high-precision orbit propagator STK HPOP.

The core of our work is to use Topex/Poseidon ephemeris as initial state vector and apply that state vector to the proposed high accurate orbit predictor. The period will be for 9.24 days to clarify the error related to real ephemeris data which is compared with the results of SGP4 and High precision orbit propagator (HPOP) module in 
system tool kit (STK), as a high precision orbit propagator trusted and used in most space agencies, which is also used to verify the attained orbital results.

Topex/Poseidon satellite will be our target for comparison for the time window 21-121992 21-00-00 UTC to 31-12-1992 02-50-00 UTC, the high accurate prediction algorithm includes (100x100) Earth Gravitational Model GM2008 released by the U.S. National Geospatial-Intelligence Agency (NGA) as high gravitational earth model [8] with solid earth tides, solar radiation pressure is then modeled using spherical shadow model [9].The Earth and the Moon are considered as eclipses bodies. Their equatorial positions are calculated using NASA jet propulsion laboratory(JPL) Ephemerides using JPL's HORIZONS system [10].The atmospheric drag Jacchia-Bowman 2008 model is used for calculating changes in density with take into account geomagnetic storm and space weather environment at each step instead of tabular values with interpolation process. The $3^{\text {rd }}$ body effect is also included and is represented by the Moon, the Sun and other planets [11].

The simulation indices for space weather solar flux F10.7,S10, M10 and Y10 and geomagnetic indices DTC1 to DTC24 for calculating density variations, Earth orientation parameters for precise calculation of transformations from International Celestial Reference Frame (ICRF) to International Terrestrial Reference Frame (ITRF) and vice versa. Nutation, precession, and polar motion are considered variables for each step in the simulation using earth orientation parameters (eop) (NGA)

\section{DYNAMIC MODEL}

As numerical methods increase exponentially in modeling over the analytical methods as computers become faster. The most attractive thing about numerical method is its ability to inject any perturbing force at any step during the simulation. The perturbed forces are added to the two-body equation which known as Cowell's formulation to get the total acceleration of satellite is expressed as:

$$
\mathbf{a}=-\frac{\mu}{r} \mathbf{r}+\mathbf{a}_{\text {perturped }}
$$

where $\mu$ is the gravitational coefficient and $\mathbf{r}$ is the satellite position vector.

The solution of the system is accomplished using variable step, variable order Adams-Bashforth-Moulton as it is more efficient more than RK45 as the function is expensive to evaluate.

\section{Gravity Model}

Since the gravity is the largest perturbing force affect the satellite as the earth is not perfect sphere so the force implied by the gravitational field is not uniform with variation in position. The asymmetric distribution of the earth to represent the gravitational potential $(U)$ as a decomposition of spherical harmonics is defined as $[12,13]$ : 


$$
U=\frac{\mu}{r}\left[1+\sum_{l=2}^{\infty} \sum_{n=0}^{l}\left(\frac{R_{\oplus}}{r}\right)^{l} P_{l, n} \sin \phi_{s a t}\left\{C_{l, n} \cos \left(n \lambda_{s a t}\right)+S_{l, n} \sin \left(n \lambda_{s a t}\right)\right\}\right]
$$

The gravitational acceleration is defined as:

where

$$
\mathbf{a}_{\text {gravity }}=-\nabla U
$$

$\begin{array}{ccl}\lambda_{\text {sat }} & \ldots & \text { The longitude of the satellite. } \\ \emptyset_{\text {sat }} & \ldots & \text { The geocentric latitude of the satellite. } \\ R_{\oplus} & \ldots & \text { Earth mean radius. } \\ \mathrm{P}_{l, \mathrm{n}} & \ldots & \text { Legendre polynomials. } \\ C_{l, \mathrm{n}} \text { and } S_{l, \mathrm{n}} & \ldots & \begin{array}{l}\text { Gravitational coefficients from the Earth } \\ \text { Gravitational Model } 2008(\mathrm{EGM} 2008) .\end{array} \\ l \text { and } n & \ldots & \text { Degree and order of the decomposition. }\end{array}$

The breakdown of the spherical harmonics as expressed in table (1).

Table 1. Gravity model parameters.

\begin{tabular}{|c|c|c|}
\hline Zonal & Sectorial & Tesseral harmonics \\
\hline$n=0$ & $I=n$ & $I \neq n \neq 0$ \\
\hline
\end{tabular}

But, the largest effect due to zonal harmonics $l=2$ which correspond to J2 representing the equatorial bulge of the earth.

\section{Atmospheric Drag}

Since LEO satellite is considered, the atmospheric drag is also dominant perturbed force acting on the satellite. It comes after gravitational especially for eccentric orbits as it tends to circulate them effect so it cannot be neglected for more precision calculations. The resulting acceleration due to drag effect is expressed as $[13,14]$ :

$$
\mathbf{a}_{\text {drag }}=-\frac{1}{2} \rho\left(\frac{C_{D} A}{m}\right) v_{r e l}^{2}
$$

where

$\begin{array}{cll}m & \ldots & \begin{array}{l}\text { Satellite mass. } \\ \mathrm{C}_{D}\end{array} \\ & \ldots & \begin{array}{l}\text { Coefficient of drag with assumption it is } \approx 2.179 \\ \text { (dimensionless) }\end{array} \\ \rho & \ldots & \text { Atmospheric density. } \\ \mathrm{A} & \ldots & \text { Area normal to } v_{\text {rel }} \\ v_{r e l} & \ldots & \text { Relative velocity to the atmosphere. }\end{array}$

The difficulties of this model are the complexity calculation of thermospheric density as it is affected by many phenomena: solar radiations, aurora, joule heating at high latitude, solar extreme ultraviolet fluxes represented by indices F10.7, Ap, and Kp. The used model in the algorithm is JM2008 as it is a combination between the high accuracy drag model developed by air force space battle lab by making analysis for 
drag on LEO satellites and the empirical density model which is used at Joint Space Operations Center (JSpOC).

\section{Third Body Effect}

The third body gravitational effect is also included in the proposed model. The acceleration due to these bodies is expressed as [15]:

where

$$
\mathbf{a}_{3 \mathrm{rd} \mathrm{body}}=\mu_{3 \mathrm{rd}}\left(\frac{\mathbf{r}_{\text {sat }, 3}}{r_{\text {sat }, 3}^{3}}-\frac{\mathbf{r}}{r^{3}}\right)
$$

$$
\begin{array}{lll}
\mu_{3 \mathrm{rd}} & \ldots & \begin{array}{l}
\text { Gravitational parameter of } 3 \mathrm{rd} \text { body planet (sun, } \\
\text { moon, ...) }
\end{array} \\
\mathrm{r}_{\mathrm{sat}, 3} & \ldots & \text { Vector from the satellite to the } 3^{\text {rd }} \text { body. }
\end{array}
$$

JPL Ephemerides are then used to calculate vectors of $3^{\text {rd }}$ planet in International Celestial Reference Frame (ICRF) at every step of the model.

\section{Solar Radiation Pressure}

Moreover, the solar radiation pressure is also included in the proposed model and is described as [12]:

$$
\mathbf{a}_{\text {srp }}=-v \frac{C_{r} A_{s}}{m} \frac{p_{s r}}{r_{\text {sat }, \text { sun }}^{3}} \mathbf{r}_{\text {sat }, \text { sun }}
$$

Where

$\begin{array}{lll}v & \ldots & \begin{array}{l}\text { The shadow function equal }(0) \text { if satellite in } \\ \text { shadow, otherwise (1) assuming geometrical } \\ \text { shadow mode including shadow of moon and } \\ \text { earth with respect to satellite. }\end{array} \\ C_{r} & \ldots & \begin{array}{l}\text { The coefficient of solar radiation }\left(1 \leq C_{r} \leq 2\right) \text { while } \\ \text { equal } 1 \text { for black body }\end{array} \\ A_{s} & \ldots & \text { Cross section area seen by sun. } \\ p_{s r} & \ldots & \text { Solar radiation pressure. }\end{array}$

\section{RESULTS AND DISCUSSION}

The proposed model is applied to TOPEX/Poseidon satellite with perigee altitude $1339.07 \mathrm{~km}$ and inclination 66.0492 degree at the epoch mentioned. The model results are then compared to both SGP4 and STK with HPOP. The STK with HPOP model used include EGM2008 for gravity model, the highest model in the program for density calculations (NRLMSISE2000), spherical model for solar radiation pressure, and dual cone for shadow model including eclipsing bodies as moon and third body effect by other planets using Bulirsch Stoer as automatic step size integrator.

Box and whisker plots of position errors for predictions using the proposed model and using STK HPOP and SGP4 are shown in Fig. 2. Minimum and maximum prediction errors are represented by the whiskers extending up and down from each box, 

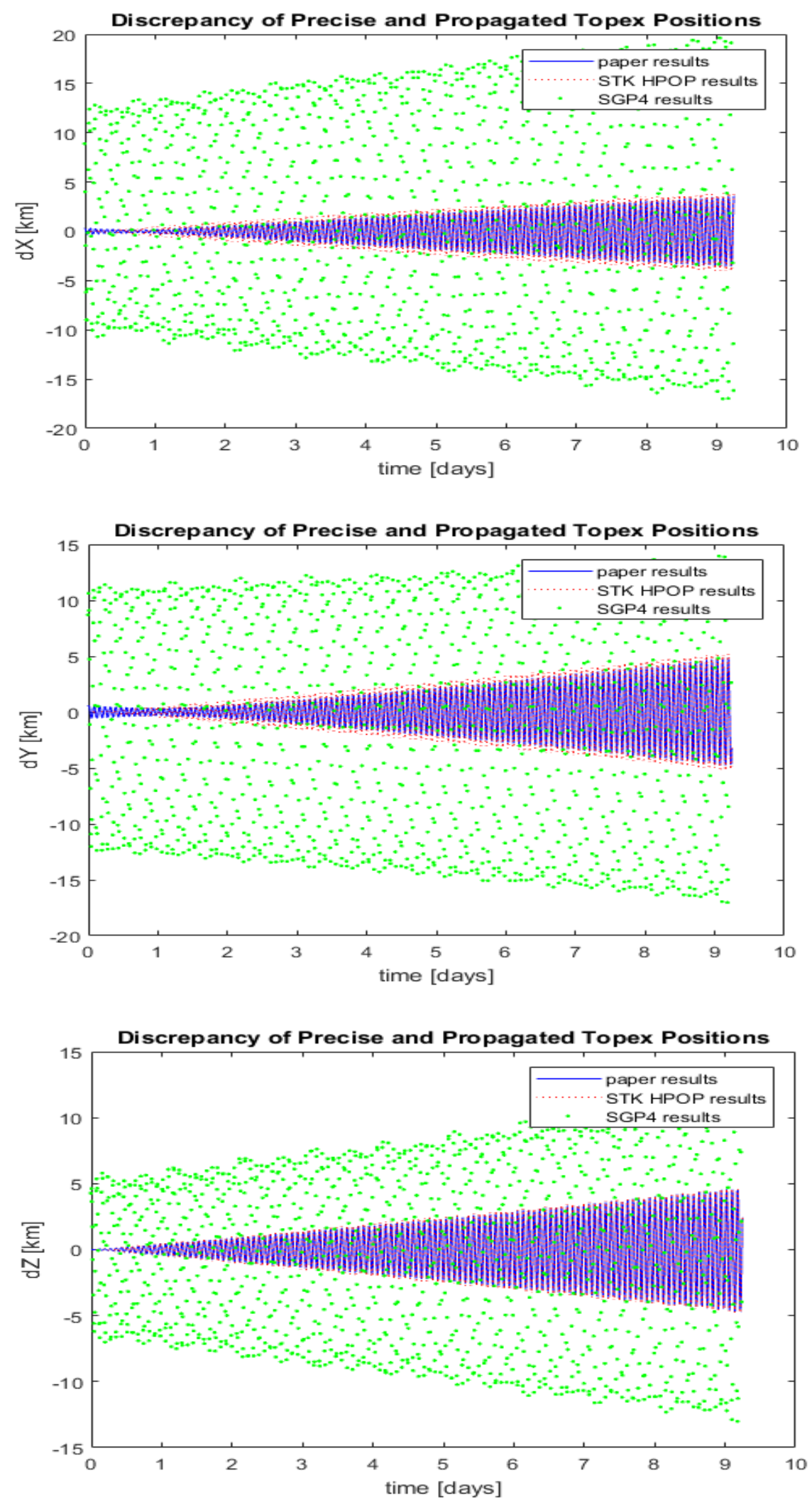

Fig.1. Position error related to POEs of Topex at the epoch mentioned with our model results, STK HPOP and SGP4at prediction period less than 10 days. 
"Box and whisker" plots of position errors for predictions using our model

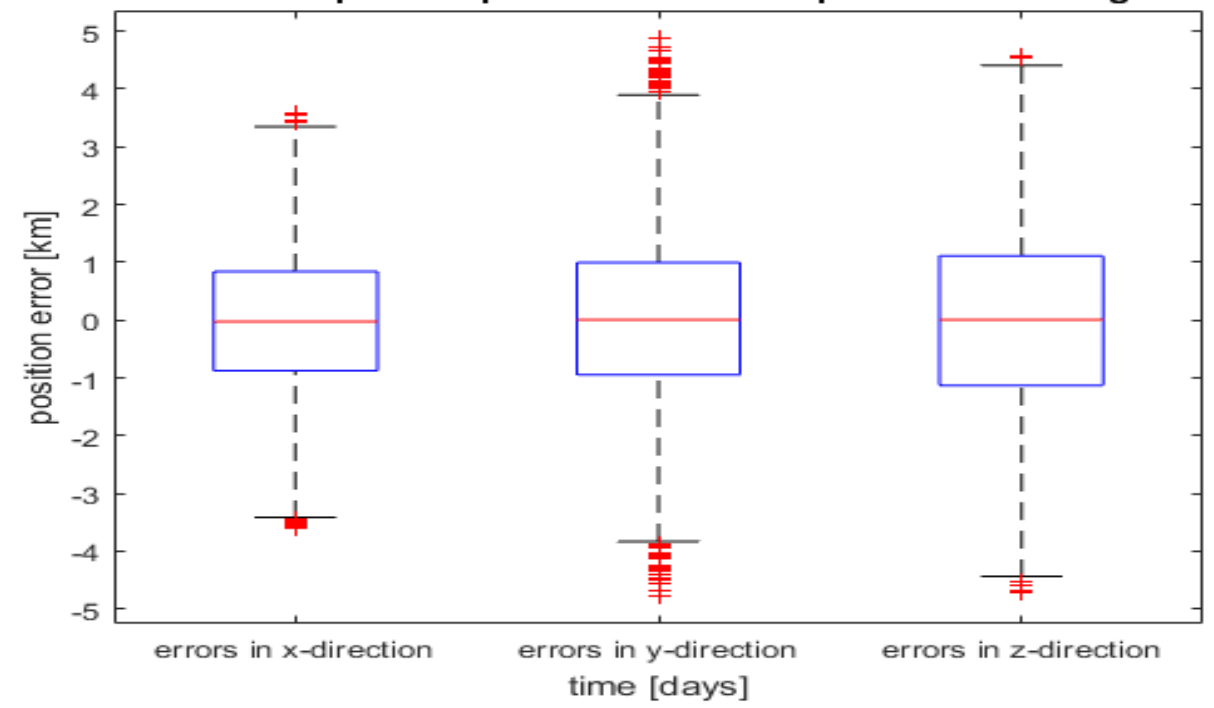

"Box and whisker" plots of position errors for predictions using STK HPOP
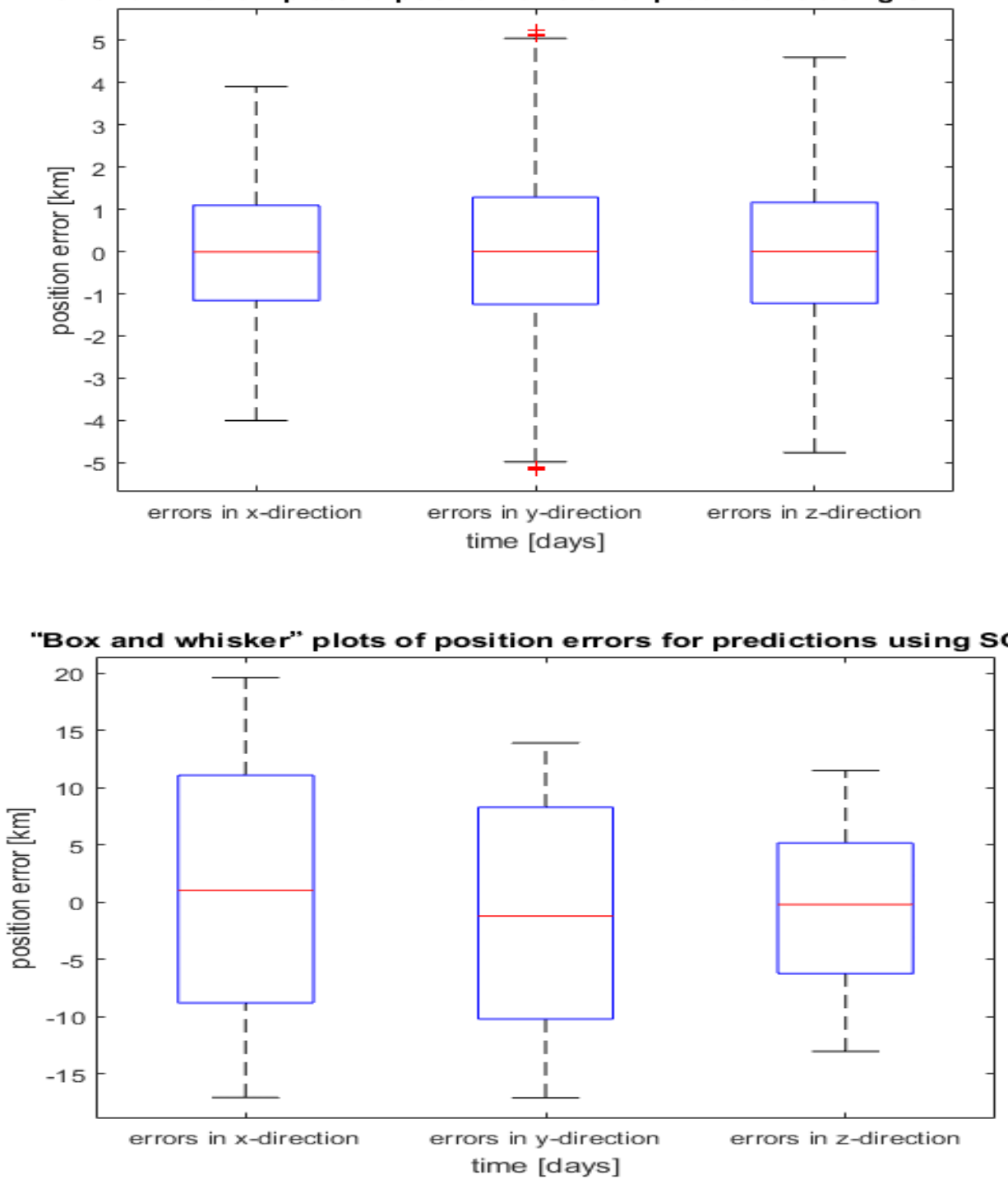

Fig. 2. "Box and whisker" plots of position errors. 
respectively. The top and low edges of the box bound the 25 th and $75^{\text {th }}$ percentiles, respectively. The line inside the boxes marks the median prediction error. All errors are plotted with respect to POEs of Topex satellite at the epoch mentioned. The proposed model makes a better result than STK HPOP as shown in Table (2)

Table 2. Comparison between position errors for the proposed model and STK HPOP model and SGP4 model related to precision orbit ephemeris.

\begin{tabular}{|c|c|c|c|}
\hline model & Error in x-axis $(\mathbf{k m})$ & Error in y-axis $\mathbf{( k m )}$ & Error in z-axis $(\mathbf{k m})$ \\
\hline Proposed model & 3.576669 & 4.878638 & 4.579291 \\
\hline STK HPOP & 3.908878 & 5.238257 & 4.597250 \\
\hline SGP4 & 19.593199 & 13.91445 & 11.48309 \\
\hline
\end{tabular}

\section{CONCLUSIONS}

The proposed model proved its ability to perform successful orbit propagation estimations providing an initial conjunction assessment accurately for a future collision avoidance system and for available data for debris. The module could be the key elements for $n$ autonomous many to many collision assessments. The exponentially increase in space objects with available TLEs as initial guess and our module as propagator are a superior solution.

Finally, obtaining the ability to perform an accurate prediction for space objects means saving resources by perform only well calculated small maneuvers and a comparative for ready recent commercial software as STK and the free online tools which uses SGP4 as the propagator for assessment collision analysis leading to nonpromising inputs for mission control center.

\section{REFERENCES}

[1] Kelso, T. (2007). "Validation of SGP4 and IS-GPS-200D against GPS precision ephemerides", $17^{\text {th }}$ AAS/AIAA Space Flight Mechanics Conference, Arizona, USA

[2] Liou, J.-C. and N. L. Johnson (2008). "Instability of the present LEO satellite populations", Advances in Space Research 41(7): 1046-1053.

[3] Levit, C. and W. Marshall (2011) "Improved orbit predictions using two-line elements", Advances in Space Research 47(7): 1107-1115.

[4] Bennett J., Sang J., Smith C. and Zhang K. (2012) "Improving low-Earth orbit predictions using two-line element data with bias correction", Advanced Maui Optical and Space Surveillance Technologies, Hawaii, USA.

[5] Muldoon A. R., Elkaim G. H., Rickard I. F. and Weeden B. (2009). "Improved orbital debris trajectory estimation based on sequential TLE Processing", IAC09. A6. 2.9, presented at the $60^{\text {th }}$ International Astronautical Congress. Daejeon, South Korea. 
[6] Easthope P. (2015). "Examination of the mean motion used in SGP4", Astrophysics and Space Science 357(2): 161.

[7] Bussy-Virat C., Getchius J. and Ridley A. (2018). "The Spacecraft Orbital Characterization Kit and its Applications to the CYGNSS Mission", 2018 Space Flight Mechanics Meeting, Florida, USA.

[8] Kenyon, S. et al. (2007). "Toward the next Earth gravitational model", SEG Technical Program Expanded Abstracts, Society of Exploration Geophysicists: 733-735.

[9] Frueh C., Jah M. and Kelecy T. (2013). "Coupled orbit-attitude dynamics of high area-to-mass ratio (HAMR) objects: influence of solar radiation pressure, Earth's shadow and the visibility in light curves", Celestial Mechanics and Dynamical Astronomy 117(4): 385-404.

[10] Nasa (2018-Jan-24). "Ephemerides", https://ssd.jpl.nasa.gov/?ephemerides.

[11] Bowman B. R.,Tobiska W. K., Marcos F., Huang C., Lin C. and Burke W. (2008), "A new empirical thermospheric density model JB2008 using new solar and geomagnetic indices", AIAA/AAS Astrodynamics Specialist Conference, AIAA, Hawaii, USA.

[12] Vallado, D. (2007). "Fundamentals of Astrodynamics and Applications", Fundamentals of Astrodynamics and Applications, Berlin: Springer, 2007. ISBN: 978-0-387-71831-6.

[13] Montenbruck, O. and E. Gill (2012). "Satellite orbits: models, methods and applications", Springer Science \& Business Media.

[14] Gurfil, P. and P. K. Seidelmann (2016). "Celestial Mechanics and Astrodynamics: Theory and Practice", Springer.

[15] Markley, F. L. and J. L. Crassidis (2014). "Fundamentals of spacecraft attitude determination and control", Springer. 